\title{
Observación frente al tratamiento electivo en cáncer de lengua móvil con cuello clínicamente negativo: resultados de una serie de 243 pacientes
}

\author{
S. Córdoba, J. Romero, F. Valcárcel, R. Magallón, C. A. Regueiro, M. I. García Berrocal, I. Zapata,
} C. de la Fuente, E. Chajón, A. de la Torre

\section{Resumen}

- Objetivo: determinar el valor del tratamiento electivo del cuello y el riesgo de enfermedad microscópica en pacientes sin adenopatías al diagnóstico (NO).

- Material y métodos: de 318 pacientes con diagnóstico histológico de carcinoma epidermoide de lengua móvil, tratados entre 1967 y 1997, 247 presentaron un estadio N0. Características: 187 varones y 56 mujeres; edad mediana: 57 años (24-89); estadios I: 69 pacientes, II: 101 pacientes, III: 39 pacientes, IV: 11 pacientes y 23 pacientes recidivas postquirúrgicas. El tumor primario fue tratado con braquiterapia (121 pacientes), irradiación externa (17 pacientes) o combinación de ambas (105 pacientes). Las áreas ganglionares se trataron con cirugía electiva en 13 pacientes, irradiación electiva en 127 pacientes (dosis mediana: $50 \mathrm{~Gy}$ ), y 103 pacientes fueron sometidos a observación. Análisis estadístico: test Kaplan-Meier y Log-rank para comparación de curvas de supervivencia.

- Resultados: la supervivencia causa específica y el control local actuarial a 5 años fue $65,3 \%$ y $66,9 \%$, respectivamente. El intervalo libre de enfermedad locorregional a 5 años fue del $52,1 \%$. La tasa de recidiva ganglionar según la modalidad de tratamiento cervical fue $0 \%, 21,3 \%$ y $25,7 \%$ para cirugía electiva, irradiación electiva y observación, respectivamente. El control ganglionar actuarial a 5 años fue para los mismos grupos del $100 \%, 78,7 \%$ y $74,3 \%$ (p=NS). Para evaluar el riesgo de enfermedad microscópica, analizamos la influencia de las variables pronósticas en el subgrupo de 93 pacientes con estadios precoces sometidos a observación. La única variable identificada como factor pronóstico independiente en el análisis multivariante fue el espesor tumoral, con un control ganglionar actuarial a 5 años del $16,7 \%$ vs. $86,7 \%$, para espesores mayores y menores de $1 \mathrm{~cm}$, respectivamente ( $p=0,0003)$.

- Conclusiones: dada la alta incidencia de recidiva ganglionar y la baja supervivencia en los pacientes con esta recidiva, consideramos aconsejable el tratamiento electivo del cuello en pacientes con tumores de lengua móvil.

Palabras clave: Lengua móvil. Ganglios cervicales. Tratamiento electivo. Radioterapia.

Oncología, 2004; 27 (1):13-20

Servicio de Oncología Radioterápica

Hospital Universitario Puerta de Hierro Madrid 


\section{S. Córdoba y cols.}

\section{Summary}

- Purpose: To determine the role of the neck elective treatment and the risk of occult lymph node metastases in clinically negative (NO) early stage mobile tongue carcinomas.

- Methods and materials: The study of 243 out of 318 patients with histologically confirmed mobile tongue carcinomas with clinically negative neck seen between 1967 and 1999 is presented. The sample consisted of 187 males and 56 females with a median age of 57 years (range, 24-89 years). According to the 2002 TNM stage classification, 69 patients belonged to stage I, 101 patients to stage II, 39 patients to stage III, and 11 patients to stage IV. The primary tumors were treated by brachytherapy alone (121 patients), external beam radiation therapy alone (17 patients) or a combination of both (105 patients). The management of the neck lymph nodes was individualized according to the characteristics of the patients: 13 were treated with elective neck dissection, 17 with elective neck irradiation (median dose $50 \mathrm{~Gy}$ ), and 103 patients were carefully followed up without any elective treatment. The statistical analysis was made by means of the Kaplan-Meyer and Logrank tests for survival curves comparison.

- Results: The five-year actuarial cause-specific survival and the local control were 65.3 and $66.9 \%$, respectively. The general five-year loco-regional disease-free survival was $52 \%$. The nodal relapse rate according to the treatment modality employed was $0 \%$ for elective neck dissection, $21.3 \%$ for elective radiation therapy, and $25.7 \%$ for the cases followed up without any elective treatment. The corresponding regional disease-free survivals were $100 \%, 78.7 \%$ and $74.3 \%$ ( $p=N S$ ). The prognostic variables offered by the subset of patients without any elective therapy were analyzed to evaluate the risk of occult node metastases. Tumor thickness was identified as a prognostic factor of regional relapse by multivariate analysis; the 5 -year regional disease-free survival was $86.7 \%$ for a thickness $<1 \mathrm{~cm}$ vs $16.8 \%$ for a thickness $>1 \mathrm{~cm}$ ( $p=0.0003$ ).

- Conclusions: Considering the high incidence of lymph node relapse and the poor survival rate of relapsing patients, we recommend the elective neck dissection to treat all patients with early stage mobile tongue carcinoma.

Key words: Mobile tongue carcinoma. Neck lymph nodes. Elective treatment. Radiotherapy.

\section{Introducción}

El cáncer de lengua móvil representa el segundo más frecuente de los cánceres de la cavidad oral, después de los de labio. En relación con otros tumores del área de cabeza y cuello, los tumores de lengua móvil tienen una alta incidencia de metástasis ganglionares regionales, entre $15-75 \%$ dependiendo del estadio inicial del tumor primario. En estadios precoces, la incidencia de enfermedad metastásica ganglionar oculta oscila entre $20-40 \%$ según las distintas series quirúrgicas ${ }^{1-3}$. Hasta un $3-5 \%$ debutan con afectación ganglionar bilateral y en un $15-20 \%$ presentan al diagnóstico metástasis ganglionares contralaterales ${ }^{4}$.

El patrón de recidiva más frecuente es locorregional y generalmente ocurre en los dos primeros años. Los tumores de lengua móvil tienen una alta propensión a la recidiva local, a pesar de tratamientos radicales. La segunda forma más frecuente de recidiva es a nivel ganglionar.
Para pacientes con cuello clínicamente negativo existen tres alternativas terapéuticas: observación estrecha, cirugía o irradiación electivas. Cualquiera de estas modalidades de tratamiento se han considerado igualmente efectivas, por lo que la elección de una u otra dependerá de la modalidad terapéutica elegida para el tumor primario. Las ventajas del tratamiento electivo serían que evitaríamos un porcentaje de recidiva cuyo rescate tiene peor pronóstico. Por otro lado, la observación permitiría evitar la morbilidad del tratamiento en un determinado número de pacientes. Sin embargo, la alta incidencia de enfermedad ganglionar subclínica, y la ausencia de factores pronósticos fiables hacen que esta alternativa deba ser utilizada prudentemente.

En el presente artículo analizamos una serie de 243 pacientes con cáncer epidermoide de lengua móvil y cuello clínicamente negativo, tratados con radioterapia. El objetivo es determinar el papel del tratamiento electivo de las áreas ganglionares frente a la observación y evaluar el riesgo de enfermedad microscópica 
ganglionar en estos últimos, con el objeto de identificar variables de carácter pronóstico que permitieran seleccionar subgrupos de pacientes que se beneficiarían de intervenciones terapéuticas más agresivas.

\section{Material y métodos}

Entre 1967 y 1997 se evaluaron y trataron con radioterapia 318 pacientes con diagnóstico histológico de carcinoma epidermoide de lengua móvil en el Servicio de Oncología Radioterápica del Hospital Universitario Puerta de Hierro. Entre los 318 pacientes 248 pacientes eran varones (78\%) y 70 eran mujeres $(22 \%)$. La relación hombres-mujeres fue de 3.5:1. La edad de los pacientes era entre 19 y 89 años con una media de 57 años.

De forma global, el $21,7 \%$ (69/318) de los pacientes tenían estadio clínico I al diagnóstico, un 31,8\% $(101 / 318)$ presentaban estadio clínico II, un $22,3 \%$ (71/318) estadio clínico III y un $14.8 \%(47 / 318)$ estadio clínico IV. El $22 \%$ de los pacientes $(70 / 318)$ presentaban un estadio $\mathrm{Tl}$, un $39 \%(124 / 318)$ estadio T2, el $19,2 \%(61 / 318)$ se estadiaron como T3 y un $10,4 \%$ eran estadios T4 (33/318). Doscientos cuarenta y tres pacientes presentaban estadio N0 $(76,4 \%)$ y 75 pacientes $(23,6 \%)$ presentaban adenopatías palpables. (Tabla I).

TABLA I

Distribución de los pacientes según el estadio tumoral Ty estadio ganglionar $\mathbf{N}$

\begin{tabular}{lrrrrrr}
\hline & N0 & N1 & N2 & N3 & & \\
\hline T1 & 69 & 1 & & & 70 & $(22,0 \%)$ \\
T2 & 101 & 17 & 6 & & 124 & $(39 \%)$ \\
T3 & 39 & 14 & 7 & 1 & 61 & $(19,2 \%)$ \\
T4 & 11 & 8 & 12 & 2 & 33 & $(10,4 \%)$ \\
Recidivas & 23 & 5 & & 2 & 30 & $(9,4 \%)$ \\
Total & 243 & 45 & 25 & 5 & 318 & \\
\hline
\end{tabular}

Entre los pacientes con cuello clínicamente negativo, 69 correspondían a un estadio T1, 101 T2, 39 T3 y 11 T4. El tumor primario fue tratado con braquiterapia exclusiva mediante implantes intersticiales con Ir192, LDR, en 121 pacientes; irradiación externa exclusiva en 17, tratamiento combinado con irradiación externa y sobreimpresión con braquiterapia en 105 pacientes. En la Tabla II se describen las características de los pacientes con cuello clínicamente negativo.
TABLA II

\section{Características de 243 pacientes con cuello clínicamente negativo}

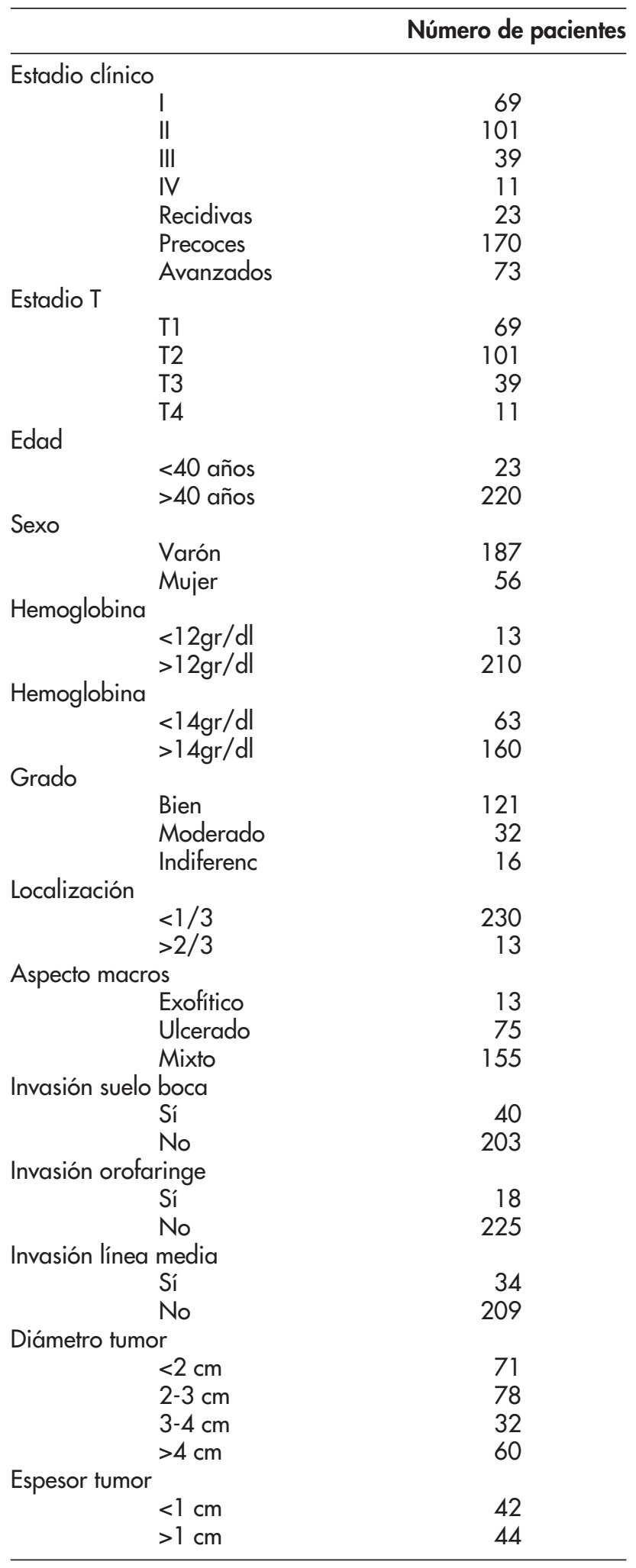




\section{S. Córdoba y cols.}

TABLA III

Análisis de supervivencia en $\mathbf{3} 18$ pacientes con carcinoma epidermoide de lengua móvil

\begin{tabular}{lccrrr}
\hline & \multicolumn{2}{c}{ Tasa bruta } & \multicolumn{2}{c}{ Tasas actuariales } \\
& \multicolumn{2}{c}{$\mathbf{5}$ años } & $\mathbf{1 0}$ años \\
\hline Supervivencia global & $202 / 318$ & $63,5 \%$ & $63.2 \%$ & $44.2 \%$ & $33.9 \%$ \\
Supervivencia causa específica & $131 / 318$ & $41,2 \%$ & $69.7 \%$ & $57.41 \%$ & $52.7 \%$ \\
Control locorregional & $170 / 318$ & $53,5 \%$ & $59.04 \%$ & $46.1 \%$ & $41 \%$ \\
Recidiva local & $121 / 318$ & $38,1 \%$ & $32.5 \%$ & $60.4 \%$ & $60.6 \%$ \\
Recidiva ganglionar & $94 / 318$ & $29,6 \%$ & $28.5 \%$ & $32.5 \%$ & $34.6 \%$ \\
\hline
\end{tabular}

El tratamiento de las áreas ganglionares, en los pacientes con cuello clínicamente negativo, incluyó la observación en 103 pacientes, 127 se trataron con irradiación cervical electiva y en 13 se realizó cirugía electiva del cuello. Entre los pacientes en los que se realizó irradiación electiva de las cadenas ganglionares de drenaje, en 46 pacientes el volumen de irradiación fue mediante 3 campos con inclusión dentro del volumen de tratamiento de todas las cadenas ganglionares, administrando mediante telecobaltoterapia una dosis mediana de 50Gy. En 81 pacientes se procedió a la irradiación, exclusivamente, de los dos tercios superiores del cuello sin irradiación de cadenas ganglionares supraclaviculares. La dosis mediana administrada fue de 45Gy (Rango 40-50Gy). De ellos, en 4 pacientes la irradiación cervical electiva se realizó mediante un campo ipsilateral de electrones que incluía los dos tercios superiores del cuello. El resto de pacientes fueron tratados con telecobaltoterapia mediante dos campos paralelos y opuestos; 5 pacientes recibieron una dosis de 20-30Gy con hipofraccionamiento y los restantes 72 se trataron mediante un fraccionamiento clásico administrando una dosis mediana de 50Gy.

\section{Resultados}

De forma general y considerando toda la serie de pacientes, la supervivencia global actuarial a 5 y 10 años respectivamente fue de $44,2 \%$ y $33,9 \%$. y la supervivencia causa específica a 5 y 10 años fue de $57,4 \%$ y $52,7 \%$, respectivamente. La tasa bruta de recidiva locorregional de la serie fue de $53,5 \%$ con un control locorregional actuarial a 5 y 10 años de $46,1 \%$ y $41 \%$, respectivamente. Ciento veintiun pacientes fracasaron inicialmente a nivel local $(38,1 \%)$ y 94 fracasaron inicialmente en las cadenas ganglionares cervicales $(29,6 \%)$. El control local actuarial fue de $44,3 \%$ a 5 años y $39,4 \%$ a 10 años. El control ganglionar actuarial a 5 y 10 años fue de $67,5 \%$ y de $65,5 \%$ respectivamente (Tabla III).

\section{Serie global: cuello clínicamente negativo}

Entre los pacientes con cuello clínicamente negativo, el intervalo libre de enfermedad locorregional a 5 años fue del $52,1 \%$. La supervivencia causa específica y el control local actuarial a 5 años fue $65,3 \%$ y $66,9 \%$, respectivamente.

Entre los 243 pacientes con cuello clínicamente negativo, 103 fueron sometidos a observación del cuello, 127 se trataron con irradiación cervical electiva y en 13 pacientes se realizó cirugía electiva del cuello. La tasa de recidiva ganglionar global fue $23,8 \%$ con un control ganglionar actuarial a dos y cinco años de $77,2 \%$ y $74,1 \%$, respectivamente. Según los estadios tumorales, el control ganglionar actuarial a cinco años fue $78,9 \%$ en los estadios $\mathrm{T} 1,81 \%$ en los estadios $\mathrm{T} 2$, $63,5 \%$ en los estadios T3 y 70,7\% en los estadios T4. Según la modalidad de tratamiento de las áreas ganglionares, el control ganglionar actuarial a dos años fue $74,3 \%$ en los pacientes sometidos a observación, $78,7 \%$ en los pacientes tratados con irradiación electiva y $100 \%$ en los tratados con cirugía electiva, respectivamente; pero estas diferencias no alcanzaron nivel de significación estadística (Fig. 1).

En el análisis univariante de influencia de las distintas variables clínico-patológicas en la recidiva ganglionar, ninguna alcanzó significación estadística salvo la edad avanzada. No obstante, al analizar el subgrupo de pacientes en los que se realizó observación del cuello, los tumores con un espesor menor de $1 \mathrm{~cm}$ presentaron un control ganglionar actuarial a cinco años de $87,5 \%$ vs. $38,1 \%$ en los tumores con un espesor mayor de $1 \mathrm{~cm}(\mathrm{p}=0,0053)$ (Fig. 2). 


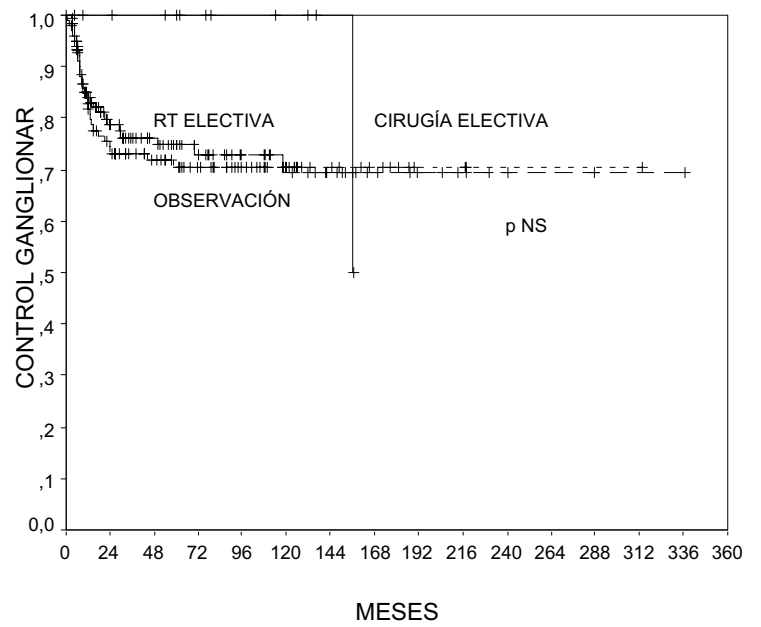

Fig. 1. Control ganglionar según la modalidad de tratamiento en pacientes con cuello clínicamente negativo.

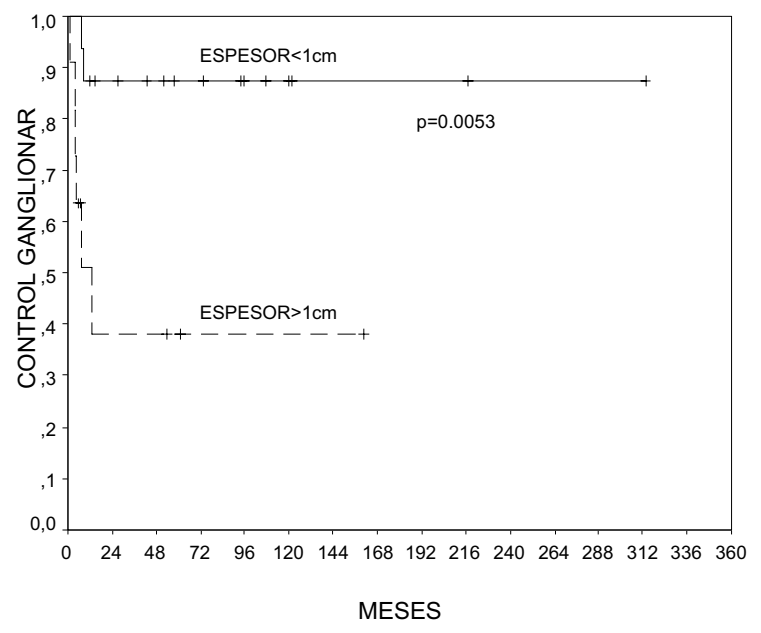

Fig. 2. Control ganglionar en 103 pacientes con cuello negativo sometidos a observación. Influencia del espesor tumoral.

\section{Estadios precoces: influencia de la modalidad de tratamiento del cuello}

Al analizar la influencia del tratamiento de las áreas ganglionares entre los 170 pacientes con estadios precoces, 92 fueron sometidos a observación del cuello y 78 recibieron tratamiento electivo (cirugía electiva en 11 pacientes e irradiación electiva en 67). La tasa de recidiva ganglionar en estadios I fue $27,5 \%$ y en estadios II $21,7 \%$, con un control ganglionar actuarial a dos años de $79 \%$ y $81 \%$, respectivamente (pNS).

Al analizar el tratamiento de las áreas ganglionares, observamos una estrecha relación tanto con el es- tadio clínico como con el tratamiento realizado sobre el tumor primario. Un 76,8\% (53/69) de los pacientes con estadio I fueron sometidos a observación del cuello frente a $61,3 \%(62 / 101)$ de los pacientes con estadio II $(p<0,001)$. Entre los pacientes en los que se realizó implante exclusivo, un $80,9 \%(89 / 110)$ fue sometido a observación del cuello frente a un $5 \%(3 / 57)$ de los pacientes tratados con irradiación externa e implante y ninguno de los 3 pacientes tratados con irradiación exclusiva $(p<0,0001)$. Debido a esta selección del tratamiento cervical ganglionar según el estadio clínico y, por tanto, según la modalidad de tratamiento del tumor primario, no es posible comparar ambos tipos de tratamiento del cuello, observación vs. tratamiento electivo, en pacientes con estadios precoces.

\section{Riesgo de enfermedad microscópica}

Noventa y dos pacientes con estadio clínico I y II (53 y 39, respectivamente) fueron sometidos a observación cervical. El control ganglionar actuarial a dos y cinco años fue respectivamente $73,8 \%$ y $69,5 \%$. La supervivencia global a cinco años fue $32,7 \%$ frente a $74,7 \%$, entre los pacientes que presentaron recidiva ganglionar frente a los que no recidivaron $(p<0,0001)$. La supervivencia causa específica a cinco años fue respectivamente $35,1 \%$ vs. $92,7 \%$ ( $p<0,0001$ ). En el análisis univariante sólo el espesor en profundidad demostró influencia significativa, con un control ganglionar actuarial a dos y cinco años de $86,7 \%$ frente a $16,7 \%$ en pacientes con tumores con un espesor inferior o superior a $1 \mathrm{~cm}$ respectivamente ( $p=0.0003$ ) (Fig. 3).

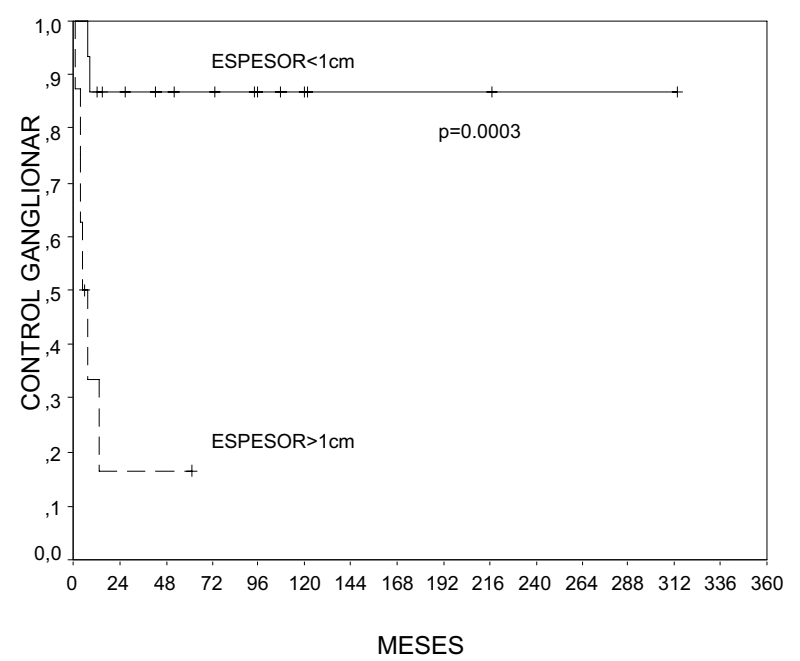

Fig. 3. Control ganglionar en pacientes con estadios precoces sometidos a observación (92 pacientes). Influencia del espesor tumoral. 


\section{S. Córdoba y cols.}

\section{Discusión}

La elección del tratamiento en pacientes con cuello negativo y especialmente en pacientes con estadios precoces, es controvertida. Entre las opciones de tratamiento se incluyen, bien la observación estrecha, bien el tratamiento electivo de las áreas ganglionares, ya sea con cirugía o con radioterapia. En nuestra serie, entre los 243 pacientes con cuello clínicamente negativo, se realizó observación en 103 pacientes, 127 se trataron con irradiación cervical electiva y 13 pacientes con cirugía electiva del cuello. No obstante, la distribución de pacientes en los tres grupos no es totalmente homogénea y los grupos no son comparables entre sí, por lo que las diferencias halladas entre los tres tipos de tratamiento podrían ser debidas más a la selección de pacientes que al propio tratamiento. La tasa de recidiva ganglionar fue respectivamente $27,2 \%, 22,8 \%$ y $7 \%$, pero estas diferencias no alcanzaron nivel de significación estadística.

En nuestra serie, al comparar la modalidad de tratamiento del cuello, no encontramos diferencias significativas entre la observación cervical vs. la irradiación - cirugía electiva de las cadenas ganglionares en los estadios precoces con un control ganglionar a dos años de $75,4 \%, 85,4 \%$ y $100 \%$ respectivamente. Aunque estas diferencias no alcanzaron significación estadística, sí encontramos una tendencia a mejor control ganglionar en pacientes en los que se realizó tratamiento electivo frente a los que fueron sometidos a observación cervical. En la literatura sólo hay dos estudios aleatorizados que comparan la observación cervical con la cirugía cervical electiva. No hay, sin embargo, ningún estudio que compare la observación con la irradiación electiva. En 1980 Vandenbrouck et al. ${ }^{5}$ publicaron el primer estudio aleatorizado comparando la observación cervical estrecha con cirugía de rescate en los pacientes que presentaban recidiva ganglionar, frente a la cirugía electiva del cuello mediante vaciamiento cervical radical ipsilateral en 75 pacientes con carcinomas de cavidad oral, T1NO, T2NO, T3NO. En este estudio no se encontraron diferencias en supervivencia entre ambas modalidades terapéuticas, por lo que se recomendaba la observación cervical y sólo cuando ésta no fuera posible recomendaban el vaciamiento cervical funcional unilateral. Sin embargo, la muestra de pacientes en cada brazo del estudio era pequeña y por otro lado los pacientes sometidos a observación presentaron una mayor incidencia, aunque no significativa, de recidiva ganglionar con extensión extracapsular, factor ampliamente reconocido por diversos autores como de peor pronóstico ${ }^{6}$. Posterior- mente, Fakih et al. ${ }^{7}$ publicaron un segundo estudio aleatorizado de 100 pacientes con estadios precoces de carcinomas de cavidad oral donde se comparaban ambas opciones, observación frente a cirugía electiva, y demostró una ventaja del $11 \%$ en supervivencia en el grupo de la cirugía electiva aunque las diferencias no alcanzaron significación estadística.

Los autores que recomiendan tratamiento electivo de las cadenas ganglionares y especialmente aquellos basados en series quirúrgicas, sustentan su opinión en base al alto índice de metástasis ganglionares ocultas, que oscilan entre $20-40 \%^{1-3,8-10}$. Las dos principales críticas al tratamiento quirúrgico electivo podrían ser, por un lado, que no es un tratamiento exento de complicaciones, con tasas de mortalidad de hasta el 2-5\% según series, y por otro lado la cirugía electiva no previene el $100 \%$ de las recidivas ganglionares ulteriores; de forma adicional, casi ningún estudio ha demostrado retrospectivamente mejora en control ganglionar y supervivencia al comparar la cirugía electiva con la observación cervical estrecha. Yuen et al. ${ }^{3}$ demostraron un $13 \%$ de recidiva ganglionar en los pacientes en los que se realizó vaciamiento cervical funcional frente al $0 \%$ en pacientes sometidos a vaciamiento cervical radical y un $48 \%$ en los pacientes sometidos a observación cervical, diferencias estadísticamente significativas, por lo que estos autores recomendaban la cirugía ipsilateral de los niveles I-II-III en estadios precoces de lengua móvil. Piedbois et al. ${ }^{11}$ compararon ambos tratamientos en 233 pacientes con estadios precoces de carcinomas de cavidad oral y aunque no demostraron diferencias en el control ganglionar entre los dos grupos, en el análisis multivariante encontraron una peor supervivencia en el grupo de observación. Sin embargo, dado que el estudio era retrospectivo y ambos grupos no estaban bien balanceados, no pudieron concluir el beneficio de uno u otro tratamiento. En esta misma línea, de forma global y sin tener en cuenta los estadios clínicos, aunque en la mayoría de las series quirúrgicas las tasas de recidiva ganglionar varían entre el 0-20\% en los pacientes sometidos a cirugía electiva frente a $20-35 \%$ en los pacientes observados, ningún estudio ha demostrado diferencias significativas entre ambas modalidades de tratamiento, quizá por el bajo número de pacientes en todos los estudios ${ }^{1,10,12,13}$.

Por otro lado, el tratamiento electivo de las cadenas ganglionares con radioterapia, ha demostrado beneficio frente a la observación cervical en algunas series y en aquellas en las que las diferencias no alcanzaron significación estadística, la tendencia era hacia una menor proporción de recidivas ganglionares en los pacientes tratados con irradiación electiva. Leborgne et al. ${ }^{14}$ analizaron 50 pacientes con carcinoma de len- 
gua móvil, TINO, T2N0 y T3NO, exentos de recidiva local. La tasa de recidiva ganglionar fue $40 \%$ frente $20 \%$ al comparar observación e irradiación electiva $(p=0,0028)$, sin encontrar diferencias según la extensión de los volúmenes cervicales irradiados. En esta línea, Wendt et al. ${ }^{15}$ no encontraron diferencias en el control ganglionar actuarial entre los pacientes observados y los tratados con irradiación electiva cubriendo sólo los niveles I, II y III. Sin embargo sí demostraron mejor control ganglionar en los pacientes en los que el volumen de radioterapia incluyó el nivel cervical IV. Meoz et al. ${ }^{16}$ demostraron también mayor beneficio de la irradiación electiva de todos los niveles frente a la irradiación limitada a los niveles I, II y III, o a la observación cervical, con una recidiva ganglionar de $24 \%$, $47 \%$ y $36 \%$ respectivamente, diferencias no significativas. Por tanto, por todo lo anteriormente expuesto, se podría concluir que existe un beneficio del tratamiento electivo en series retrospectivas frente al de observación, aunque no existan datos concluyentes derivados de estudios aleatorizados.

Aunque la mayoría de las series han fallado en demostrar beneficio del tratamiento electivo frente a la observación cervical de forma significativa, casi todos los estudios publicados demuestran un beneficio marginal del tratamiento electivo, cirugía o radioterapia, frente a la observación. Por otro lado, la recidiva ganglionar representa en cierto modo el espejo de tumores con carácter de especial agresividad, pues en general los pacientes que fracasan a nivel ganglionar tienen peor supervivencia a pesar de los tratamientos de rescate. Por ello sería de interés conocer qué variables podrían ser predictivas de recidiva ganglionar, reflejo de enfermedad microscópica a nivel cervical y actuar sobre estos pacientes con tratamientos más agresivos. En nuestra serie, entre los pacientes que presentaron recidiva ganglionar, la supervivencia global a cinco años fue $22,8 \%$ frente a $62,6 \%$ entre los pacientes que no presentaron recidiva ganglionar $(p=0,0008)$. La supervivencia causa específica a cinco años fue $31,2 \%$ vs. $76,1 \%$ respectivamente $(p<0,0001)$. El espesor tumoral clínico mayor de $1 \mathrm{~cm}$ demostró en nuestra serie clara influencia en la recidiva ganglionar tanto en el análisis univariante como en el multivariante. El hallazgo del espesor tumoral como factor pronóstico y predictivo de la enfermedad microscópica ganglionar se ha descrito por otros autores $2,9,17-21$. Sin embargo, el espesor tumoral crítico a partir del cual aumenta el riesgo de enfermedad subclínica ganglionar varía, de unos estudios a otros, entre 2 y 10 milímetros. Parece más o menos uniforme entre las distintas series recomendar tratamiento electivo en tumores con un espesor en profundidad mayor de 10 milímetros; sin embargo, no hay acuerdo general sobre el espesor mínimo a partir del cual no estaría recomendado ningún tratamiento electivo, pudiendo someter a estos pacientes a una observación estrecha.

\section{Conclusiones}

La recidiva ganglionar cervical en pacientes con carcinoma epidermoide de lengua móvil implica un pronóstico sombrío con un difícil rescate. Basados en nuestra serie $y$ en otras publicadas, parece aconsejable realizar tratamiento electivo del cuello en pacientes con cáncer de lengua móvil reservando la observación tumoral para pacientes muy seleccionados, con espesores tumorales clínicos pequeños, probablemente menores de $10 \mathrm{~mm}$. En este sentido la aportación de las técnicas diagnósticas de las que disponemos en la actualidad: ecografía, resonancia magnética y/o tomografía por emisión de positrones, permitirían evaluar el espesor tumoral a nivel local y/o la existencia de enfermedad clínicamente no evidente a nivel cervical. Esto contribuiría a la selección del tratamiento más adecuado (electivo frente a observación) para cada paciente, proporcionando las mejores probabilidades de control del cáncer de lengua móvil con la mínima morbilidad en cada situación clínica. Son necesarios estudios aleatorizados, bien diseñados, que aclaren definitivamente el valor del tratamiento electivo del cuello en pacientes con cáncer de lengua móvil.

Correspondencia:

Dra. S. Córdoba Largo

C/ Esparta, 61 - 2 D

28230 Las Rozas (Madrid)

E-mail: soficordoba@yahoo.es 


\section{S. Córdoba y cols.}

\section{Bibliografía}

1. Persky MS, Lagmay VM. Treatment of the clinically negative neck in oral squamous cell carcinoma. Laryngoscope 1999; 109:1160-4.

2. Charoenrat P, Pillai $G$, Patel $S$, et al. Tumour thickness predicts cervical nodal metastases and survival in early oral tongue cancer. Oral Oncol 2003; 39:386-90.

3. Yuen AP, Lam KY, Chan AC, et al. Clinico pathological analysis of elective neck dissection for NO neck of early oral tongue carcinoma. Am J Surg 1999; 177:90-2.

4. Lindberg RD, Fletcher $\mathrm{GH}$. The role of irradiation in the management of head and neck cancer: analysis of results and causes of failure. Tumori 1978; 64:31325.

5. Vandenbrouck C, Sancho-Garnier H, Chassagne D, et al. Elective versus therapeutic radical neck dissection in epidermoid carcinoma of the oral cavity: results of a randomized clinical trial. Cancer 1980; 46:386-90.

6. Myers JN, Greenberg JS, Mo V, et al. Extracapsular spread. A significant predictor of treatment failure in patients with squamous cell carcinoma of the tongue. Cancer 2001; 92:3030-6.

7. Fakih AR, Rao RS, Borges AM, et al. Elective versus therapeutic neck dissection in early carcinoma of the oral tongue. Am J Surg 1989; 158:309-13.

8. Byers RM, El Naggar AK, Lee YY, et al. Can we detect or predict the presence of occult nodal metastases in patients with squamous carcinoma of the oral tongue? Head Neck 1998; 20:138-44.

9. Yuen AP, Lam KY, Wei WI, et al. A comparison of the prognostic significance of tumor diameter, length, width, thickness, area, volume, and clinicopathological features of oral tongue carcinoma. Am J Surg 2000; 180:13943.

10. Beenken SW, Krontiras H, Maddox WA, et al. T1 and T2 squamous cell carcinoma of the oral tongue: prognostic factors and the role of elective lymph node dissection. Head Neck 1999; 21:124-30.

11. Piedbois P, Mazeron JJ, Haddad E, et al. Stage I-II squamous cell carcinoma of the oral cavity treated by iri- dium-192: is elective neck dissection indicated? Radiother Oncol 1991; 21:100-6.

12. Al Rajhi NM, Khafaga YM, Saleem M, et al. A study comparing different approaches in managing neck nodes in early carcinoma of the tongue. Saudi Med J 2002; 23:1343-6.

13. Benk V, Mazeron JJ, Grimard L, et al. Comparison of curietherapy versus external irradiation combined with curietherapy in stage II squamous cell carcinomas of the mobile tongue. Radiother Oncol 1990; 18:339-47.

14. Leborgne F, Leborgne JH, Barlocci LA, et al. Elective neck irradiation in the treatment of cancer of the oral tongue. Int J Radiat Oncol Biol Phys 1987; 13:1 149-53.

15. Wendt CD, Peters $L$, Delclos $L$, et al. Primary radiotherapy in the treatment of stage I and II oral tongue cancers: importance of the proportion of therapy delivered with interstitial therapy. Int J Radiat Oncol Biol Phys 1990; 18:1287-92.

16. Meoz RT, Fletcher GH, Lindberg RD. Anatomical coverage in elective irradiation of the neck for squamous cell carcinoma of the oral tongue. Int J Radiat Oncol Biol Phys 1982; 8:1881-5.

17. Woolgar JA, Scott J. Prediction of cervical lymph node metastasis in squamous cell carcinoma of the tongue/ floor of mouth. Head Neck 1995; 17:463-72.

18. Spiro RH, Huvos AG, Wong GY, et al. Predictive value of tumor thickness in squamous carcinoma confined to the tongue and floor of the mouth. Am J Surg 1986; 152:345-50.

19. Mohit-Tabatabai MA, Sobel HJ, Rush BF, et al. Relation of thickness of floor of mouth stage I and II cancers to regional metastasis. Am J Surg 1986; 152:351-3.

20. Shingaki S, Suzuki I, Nakajima T, et al. Evaluation of histopathologic parameters in predicting cervical lymph node metastasis of oral and oropharyngeal carcinomas. Oral Surg Oral Med Oral Pathol 1988; 66:683-8.

21. Al Rajhi N, Khafaga Y, El Husseiny J, et al. Early stage carcinoma of oral tongue: prognostic factors for local control and survival. Oral Oncol 2000; 36:508-14 